\title{
Nada nuevo qué contar: la irrelevancia de los aportes a la teoría de las relaciones internacionales en América Latina
}

\section{Javier Garay* Miguel Martínez**}

\section{RESUMEN}

Después de participar en muchos congresos de Relaciones Internacionales en los últimos diez ańos en América Latina, es recurrente el tema de si existe o debe existir un punto de vista latinoamericano de las relaciones internacionales. En este artículo se muestra que esta insistencia en los congresos no es algo que se refleje en la creación de conocimiento sobre la disciplina, puesto que la producción científica no muestra dicha tendencia. Por otro lado, se demuestra que esa insistencia, que resulta casi una obsesión y, por tanto, una necesidad, no es tal, puesto que el área geográfica no debe ser el factor fundamental que determine la creación de conocimiento.

Palabras clave: teoría de las relaciones internacionales, América Latina, autonomía, pensamiento.

\footnotetext{
* Doctor en Ciencia Política. Docente Investigador cipe, Facultad de Finanzas, Gobierno y Relaciones Internacionales, Universidad Externado de Colombia, Bogotá (Colombia). javier.garay@uexternado.edu.co

** Doctor en Ciencia Política y Relaciones Internacionales. Docente Investigador CIPE, Facultad de Finanzas, Gobierno y Relaciones Internacionales, Universidad Externado de Colombia, Bogotá (Colombia). miguel.martinezg@uexternado.edu.co
}

Recibido: 3 de diciembre de 2015 / Modificado: 8 de febrero de 2016 / Aceptado: 1 de abril de 2016 Para citar este artículo

Garay, J. y Martínez, M. (2016). Nada nuevo qué contar: la irrelevancia de los aportes a la teoría de las relaciones internacionales en América Latina. OASIs, 23, 31-52. DOI: http://dx.doi.org/10.18601/16577558.n23.03 
Nothing new to say:

the irrelevance of the

contributions from Latin

America to the study of

international relations

theory

\section{ABSTRACT}

The debate over whether there should be a Latin American point of view in the field of International Relations has been a common theme at International Relations conferences in Latin America in the past ten years. Nevertheless, this article aims to show that this insistence of bringing up this issue at conferences is something that has not been reflected in the creation of knowledge, since no trend can be found in the scientific production. Furthermore, this article shows that there is no need for a Latin American point of view of International Relations, since geography should not be the primary factor determining the creation of knowledge.

Key words: International relations theory, Latin America, autonomy, thought.

\section{INTRODUCCIÓN}

En cada Congreso sobre Relaciones Internacionales (RI) que tiene lugar en América Latina, se resalta la necesidad de que la región aporte una visión propia sobre los temas internacionales. De manera más reciente, incluso, se ha señalado la posibilidad de articular aportes en el ámbito teórico de esta disciplina.

Los nombres de Carlos Escudé y Roberto Russell, con su debate y sus propuestas de principios de la década de los noventa, aparecen de manera recurrente. El primero, con su propuesta de realismo periférico (Escudé, 1991; 1992, 1995) y la respuesta del segundo denominada neoidealismo periférico (Russell, 1991). En ambos casos, la idea está enfocada en guiar la política exterior del Gobierno argentino, pero no supone, en ningún caso, una alternativa teórica a lo avanzado hasta ahora en la teoría de relaciones internacionales ${ }^{1}$.

No obstante, son los únicos intentos de aportes teóricos conocidos en la región. Tal vez por ello se han realizado esfuerzos académicos que permitan rastrear o dar lugar a nuevos aportes. Uno de ellos es la revista Cuadernos de pensamiento crítico latinoamericano, del Consejo Latinoamericano de Ciencias Sociales (CLACso).

Es decir, por un lado se plantea la necesidad de una visión específicamente latinoamericana de temas internacionales; por el otro, no existen mayores ejemplos que permitan demostrar su existencia o validez teórica. De esta tensión surge la pregunta acerca de si realmente es diferente la visión latinoamericana a la ya existente en el cuerpo de teorías de relaciones internacionales. Este artículo busca demostrar que, al contrario de las expectativas,

\footnotetext{
1 Los estudios de política exterior son una parte importante de las investigaciones de relaciones internacionales, pero no son un aporte teórico comprehensivo de los asuntos estudiados por esta disciplina, que son más amplios que el comportamiento internacional de los Estados.
} 
una visión teórica propia latinoamericana ni existe, ni es necesaria.

Sin embargo, se ha creado una idea según la cual parecería necesaria una alternativa de origen latinoamericano para comprender el escenario internacional. Es más, algunos académicos extienden esta necesidad a cada una de las regiones del mundo (Tickner y Wæver, 2009; Tickner y Blaney, 2013). Esta forma de pensar implicaría que el conocimiento está delimitado por cuestiones geográficas y no por fronteras disciplinares.

Lo anterior no quiere decir que se nieguen las diferencias culturales o que no se reconozcan las especificidades de cada sociedad. Sin embargo, el reconocerlas no implica que, automáticamente, la explicación de los fenómenos de una disciplina como la internacional dependa de esas diferencias. Si así fuera, estas diferencias no se detendrían en el plano regional -al fin y al cabo construcciones artificiales-, sino que tendría que demostrarse por qué estas no llegan al plano de los países, de las ciudades o, incluso, de regiones fronterizas, por ejemplo.

Para indagar por las razones de esa percibida necesidad y supuesta diferencia en la forma como los internacionalistas latinoamericanos abordan el aspecto teórico de las relaciones internacionales, este documento hace una indagación sobre lo que se ha publicado en AL desde el punto de vista teórico.

Para demostrar la hipótesis planteada, el artículo está dividido en las siguientes secciones. Primero, se describe la metodología utilizada. Segundo, se resumen los resultados cuantitativos. Tercero, se hace un análisis de los escritos latinoamericanos sobre aspectos teóricos. Cuarto, se hace una reflexión sobre la supuesta necesidad de la visión regional. Al final se aporta una conclusión.

\section{METODOLOGÍA}

Para encontrar si existe o no un pensamiento específico, se pueden utilizar diferentes caminos. Uno de ellos es el estudio de lo publicado y, a través de técnicas de análisis del discurso, identificar la perspectiva teórica implícita en las publicaciones. No obstante, esta vía es muy amplia y, por tanto, desborda los límites en espacio y profundidad de este documento.

Por esta razón, la forma como se abordó la indagación por la pregunta fue a través de la búsqueda de publicaciones periódicas y especializadas sobre RI que existieran en los países latinoamericanos. De estas publicaciones se escogieron aquellos artículos que abordaran, de una manera u otra, cuestiones teóricas.

Una primera observación que salta a la vista es que, a pesar de existir facultades de relaciones internacionales o de temas afines en todos los países latinoamericanos, las publicaciones académicas sobre el tema no son muy numerosas. Por ello, la búsqueda se restringió, por inexistencia de material, a pocos países. Las revistas escogidas pertenecen a México, Argentina, Ecuador, Perú, Venezuela, Chile, Brasil y Colombia.

La observación de artículos se limitó a los años comprendidos entre 2006 y 2014. Esta decisión se debió, primero, a una cuestión profesional. Los autores asistieron a Congresos latinoamericanos de RI en este periodo de tiempo. Asimismo, casi una década es suficiente para establecer, por un lado, si hay aportes 
teóricos propiamente latinoamericanos y, por el otro, si existe inquietud real de los académicos de la región por crear estos aportes.

Dentro de los documentos incluidos en el estudio solo se tuvieron en cuenta los artículos académicos. Las reseñas de libros fueros excluidas debido a que estas no demuestran los aportes de quien las escribe. El análisis de artículos permite identificar tendencias, al provenir de publicaciones periódicas, debates teóricos entre autores y las principales inquietudes en este ámbito. De hecho, los autores mencionados en la introducción -Escudé y Russell- hicieron sus aportes, inicialmente, en publicaciones de estas características y, además, como resultado de un debate entre los dos.

Después de la identificación de los escritos teóricos se hizo una lectura crítica de los mismos para señalar las diferencias entre estos y las teorías de relaciones internacionales que no son consideradas latinoamericanas. De esta manera, se pudo identificar qué tan latinoamericanos son los aportes hechos. Ca- be aclarar que todos los resultados, el análisis posterior y la conclusión, se refiere únicamente al contenido de lo investigado, es decir, las revistas revisadas por los autores, por lo que si existe algún aporte a la teoría de relaciones internacionales en algún libro u otro medio, no está considerado en este artículo.

A continuación, se presentan los resultados cuantitativos del estudio.

\section{RESULTADOS CUANTITATIVOS}

En la tabla 1 se resumen los hallazgos de la revisión de ocho publicaciones periódicas académicas en temas internacionales de cinco países representativos de la región. La búsqueda de las publicaciones se hizo por medio de las bases de datos Red de Revistas Científicas de América Latina, el Caribe, Espańa y Portugal (Redalyc) y Sistema Regional de Información en Línea para Revistas Científicas de América Latina, el Caribe, España y Portugal (Latindex). Una lista extensa de las revistas inclui-

CUADRO 1. TOTAL DE ARTÍCULOS SOBRE TEORÍA DE RELACIONES INTERNACIONALES POR PAÍS

\begin{tabular}{|l|c|c|c|c|c|c|c|c|c|c|c|c|}
\hline & 2014 & $\mathbf{2 0 1 3}$ & $\mathbf{2 0 1 2}$ & $\mathbf{2 0 1 1}$ & $\mathbf{2 0 1 0}$ & $\mathbf{2 0 0 9}$ & $\mathbf{2 0 0 8}$ & $\mathbf{2 0 0 7}$ & $\mathbf{2 0 0 6}$ & Total artículos & Total TRI & Porcentaje \\
\hline Argentina & 118 & 57 & 52 & 54 & 62 & 55 & 65 & 45 & 51 & 559 & 2 & 0,36 \\
\hline Brasil & 224 & 272 & 220 & 169 & 155 & 94 & 91 & 62 & 23 & 1330 & 39 & 2,93 \\
\hline Chile & 13 & 13 & 13 & 14 & 13 & 13 & 16 & 17 & 36 & 148 & 3 & 2,03 \\
\hline Colombia & 44 & 34 & 23 & 26 & 29 & 28 & 25 & 22 & 30 & 261 & 2 & 0,77 \\
\hline Ecuador & 25 & 27 & 26 & 31 & 36 & 33 & 29 & 32 & 36 & 275 & 0 & 0,00 \\
\hline México & 131 & 127 & 117 & 98 & 112 & 109 & 111 & 111 & 76 & 992 & 15 & 1,51 \\
\hline Perú & 5 & 7 & 8 & 11 & 10 & 10 & 10 & 30 & 14 & 105 & 0 & 0,00 \\
\hline Venezuela & 13 & 6 & 14 & 12 & 12 & 5 & 6 & 12 & 12 & 92 & 4 & 4,35 \\
\hline & & & & & & & & \multicolumn{2}{|c|}{ Totales } & 3762 & 65 & 1,73 \\
\hline
\end{tabular}


das en el estudio y de los países en donde se publican se encuentran en el Anexo 1.

De la tabla se pueden extraer las siguientes observaciones. Lo primero que llama la atención es la poca cantidad de publicaciones relacionadas con las teorías de relaciones internacionales. De un total de 3762 artículos publicados entre el periodo 2006-2014, tan solo 65 fueron sobre este tema, lo que equivale a un $1,73 \%$ del total. No es dable esperar contribuciones originales provenientes de la región, si no hay publicaciones sobre el tema teórico.

En segundo lugar, se puede observar que México, Brasil y Chile, en ese orden, son los países que, en términos proporcionales, más publican artículos de carácter teórico. Si se tiene en cuenta que la mayoría de autores están de acuerdo en considerar a estos países como potencias regionales, podría esta observación ser evidencia para futuras investigaciones que relacionen el carácter de potencia de un país con un mayor interés en los temas internacionales, comenzando por el aspecto teórico.

Una tercera observación tiene que ver con el aspecto temporal. No existe una tendencia clara de un ańo o grupo de ańos en los que más se hayan publicado artículos de carácter teórico. Esto podría indicar que lo publicado no forma parte de un esfuerzo de reflexión teórica consistente ni sostenido en el tiempo en la región, sino que los artículos resultan de investigaciones esporádicas. Esto, a pesar de los cambios en el ámbito internacional, los eventos que suscitan debates teóricos, el papel de América Latina en esos hechos y, por último, la insistencia en la supuesta necesidad de una reflexión autónoma y propia de la región.
Unido a lo anterior, una observación adicional que no surge directamente de la tabla 1 consiste en la identificación de los autores que han escrito artículos sobre temas teóricos. De todos los países y las publicaciones analizados, tan solo en Chile y en México un autor ha escrito más de un artículo sobre teoría. En el caso chileno, Pablo Anzaldi escribió dos artículos en el periodo analizado. En México, Carlos Ballesteros publicó el mismo número de artículos.

A partir de estos resultados y observaciones se encuentra evidencia para afirmar que no hay una reflexión sobre teoría de relaciones internacionales en la región latinoamericana. No obstante, esta evidencia no dice nada sobre si, tal vez, alguno de esos pocos artículos publicados tenga las características para poder considerarlo como una demostración de la existencia de un pensamiento propio latinoamericano. Para ello, es necesario profundizar en la lectura crítica de cada uno de los 18 artículos identificados. A esto se dedica el próximo apartado.

\section{UNA LECTURA CRÍTICA}

¿Cómo calificar la originalidad de un artículo de carácter teórico? Muchos podrían ser los criterios de análisis para identificar si los documentos estudiados proponen una visión latinoamericana de la teoría de las relaciones internacionales.

Si se observa, el problema planteado en el párrafo anterior comprende dos dimensiones/niveles de análisis. El primero consiste en la determinación de criterios para la identificación de propuestas originales en el 
ámbito teórico. El segundo radica, a su vez, en la designación de criterios que permitan establecer si esa originalidad tiene que ver con el contexto geográfico, cultural y social del ámbito latinoamericano.

Esto último resulta una tarea mucho más compleja de lo que a simple vista se percibe. Parte de la dificultad se encuentra en que aquellos teóricos, principalmente críticos, de las relaciones internacionales, hacen referencia a la posibilidad de la existencia de diversas epistemologías, ontologías y procesos de acumulación del conocimiento científico, pero no han dado pistas de cómo estos se pueden materializar. Por ejemplo, los pospositivistas señalan que el positivismo puede ser solo una forma de construir conocimiento. Sin embargo, no han aportado señales de qué otra metodología puede suplir la misma función.

Además de esto, se vuelve complejo señalar criterios para identificar lo que es producto de una región como la latinoamericana si se tiene en cuenta que la misma (¿in?)definición de la región resulta problemática y confusa. ¿El criterio es la nacionalidad del autor?, ¿la temática que trata?, ¿el lenguaje que usa? Cualquiera de estos posibles criterios requeriría de un esfuerzo mayúsculo de definición. Por ejemplo, si se considera que la respuesta está en el lenguaje que se usa, tendría que comenzar por delimitarse claramente cuál es el lenguaje "típicamente" latinoamericano. Y así con cada criterio.
Como el objetivo de este artículo es principalmente cuestionar creencias y explorar evidencia, se ha decidido dejar de lado la designación de criterios que definan la latinoamericanidad, más allá del origen de la publicación y de la nacionalidad de los autores.

Por su parte, del lado del nivel de la identificación de la originalidad de la propuesta, se decidió asumir como tal cualquiera que incluyera una visión alternativa o diferente frente a lo ya existente en las teorías de relaciones internacionales si el documento aportaba avances en, por lo menos, dos de cinco criterios $^{2}$, señalados en la tabla 2 .

El primer criterio se estableció debido a que una visión original debe tener en cuenta lo ya existente tanto en los vacíos de las teorías que podrían denominarse del mainstream de relaciones internacionales y aportar críticas diferentes a las que ya se han hecho en otras latitudes.

Los criterios dos y tres responden a la necesidad de cualquier propuesta teórica de establecer variables específicas y supuestos concretos que permitan utilizar el método científico para falsear o verificar hipótesis que se deriven de la aplicación de los modelos.

El criterio de temáticas incluye la presunción de que al considerarse necesaria la existencia de un pensamiento propio por región, esto se puede deber a que la pertenencia a una región específica hace que sus académicos sean conscientes de la existencia de realidades que

2 Estos criterios fueron definidos a través de discusiones entre los autores de este texto a partir de lo observado en documentos que, en su momento, fueron considerados como aportes originales a las teorías de relaciones internacionales. Dentro de esos documentos, el más estudiado fue el famoso artículo de Alexander Wendt, "Anarchy is What States Make of It: The Social Construction of Power Politics". 
CUADRO 2. CRITERIOS DE IDENTIFICACIÓN DE ORIGINALIDAD

\begin{tabular}{|l|l|}
\hline \multirow{2}{*}{ Nombre } & \multicolumn{1}{|c|}{ Preguntas orientadoras } \\
\hline \multirow{2}{*}{$\begin{array}{l}\text { Visión } \\
\text { crítica }\end{array}$} & $\begin{array}{l}\text { ¿Plantea vacíos teóricos de las propuestas ya } \\
\text { existentes? }\end{array}$ \\
\cline { 2 - 2 } Variables & $\begin{array}{l}\text { ¿Aporta críticas diferentes a las provenientes } \\
\text { de otras regiones o teorías? }\end{array}$ \\
\cline { 2 - 2 } & $\begin{array}{l}\text { ¿Aporta variables independientes novedo- } \\
\text { sas? }\end{array}$ \\
\cline { 2 - 3 } ¿Aporta variables dependientes novedosas? & ¿Aporta variables de control novedosas? \\
\hline \multirow{2}{*}{ Supuestos } & $\begin{array}{l}\text { ¿Parte de supuestos diferentes a los conven- } \\
\text { cionales? }\end{array}$ \\
\hline \multirow{2}{*}{ Temáticas } & $\begin{array}{l}\text { ¿Propone la ampliación de la agenda de } \\
\text { investigación a "otras realidades" no observa- } \\
\text { das anteriormente? }\end{array}$ \\
\hline \multirow{2}{*}{$\begin{array}{l}\text { Metodo- } \\
\text { logía }\end{array}$} & $\begin{array}{l}\text { ¿Utiliza una metodología alternativa a la } \\
\text { positivista? }\end{array}$ \\
\cline { 2 - 2 } & $\begin{array}{l}\text { ¿Utiliza una metodología alternativa a las } \\
\text { esbozadas por las teorías críticas, feministas } \\
\text { y otras? }\end{array}$ \\
\hline
\end{tabular}

Fuente: elaborado por los autores.

no han sido tenidas en cuenta por sus contrapartes en otros contextos sociales o culturales.

Por último, el criterio metodológico incluye la discusión adelantada por la visión de los autores críticos y de los pospositivistas según la cual el método científico occidental ha impedido la evolución de otros métodos. Si se considera necesaria la existencia de un pensamiento latinoamericano -en oposición al occidental establecido-, podría darse lugar a la propuesta de metodologías alternativas.

En este orden de ideas, el análisis profundo de los 65 artículos identificados como de teoría arroja los siguientes resultados. Después de la primera lectura, se encontró que, de ma- nera general, estos podrían clasificarse en dos grandes grupos. Por un lado, están aquellos que no buscan proponer ninguna visión alternativa sino que son meramente descriptivos de alguna teoría ya existente o que intentan aplicarla. Por el otro, están los artículos que pretenden proponer algún avance teórico. De los 65 artículos, 52 se encuentran en la primera categoría y los trece restantes en la segunda. De esta manera, se reduce aún más la producción intelectual autónoma y original de los autores latinoamericanos en materia teórica.

Los artículos meramente descriptivos abordan temas como los avances y aportes de las teorías de relaciones internacionales (Cid, 2008), la aplicación del realismo a toma de decisiones (Dallanegra, 2009), constructivismo (Sánchez, 2012), evolución histórica de las teorías (Murillo, 2013; De Oliveira y Geraldella, 2013; de Melo Santos; 2012; Pereira y Assumpcao, 2014), conceptos en relaciones internacionales (Cervo, 2008; Faria, 2013; Ramos, 2013; Malamud, 2012; Sozzi, 2013; Lozurdo, 2013; Aguiar, 2011; Rodríguez, 2014; Scherma, 2012), concepto de autonomía en el realismo (Bernal, 2013), escuela inglesa (Sombra, 2006; Maione de Souza, 2008 y 2013; Treinto, 2008; Alves Ribeiro, 2013; Dutra, 2015), Raymond Aron y Clausewitz (Anzaldi, 2008; 2009), aplicación de la filosofía de Jacques Maritain (Papini, 2009), constructivismo realista desde la periferia (Becerra, 2013) y aplicación del posmodernismo y del constructivismo a la política exterior colombiana (Carvajal, 2009).

De igual manera, varios artículos hacen un recorrido por la teoría de la dependencia, el estructuralismo y autores semejantes (Bac- 
chi, 2013; Tello, 2010; Sandrím, 2011; Ramos, 2013; Florencio, 2015; Ramos, 2012; Castro, 2008; Gallardo y Hassoun, 2014; Quintero, 2014; Fernández, 2014; Prada y Quintero, 2014).

Aunque se sale del objeto de estudio de este documento, como es evidente en algunas de las aplicaciones de conceptos o de teorías seńaladas, en la investigación se hizo patente una concentración mayoritaria de los escritos internacionales en América Latina enfocada en los temas de política exterior de los países. Por ello, la perspectiva teórica, cuando se aplica, se hace en esta subárea de las RI.

Tanto los artículos meramente descriptivos como aquellos que buscan hacer aportes a la teoría presentan las siguientes características comunes.

Primero, aunque se anuncie la intención de hacer aportes teóricos, los autores se limitan a realizar descripciones históricas de la evolución de la disciplina, del ámbito internacional o de algunos conceptos (Romero, 2007; Vanegas, 2007; Sosa, 2006; Ballesteros, 2007; 2012; Cid, 2008; Murillo, 2013; Cervo, 2008; Faria, 2013; Ramos, 2013; Malamud, 2012; Sozzi, 2013; Lozurdo, 2013; F. de Oliveira y Geraldella, 2013; Melo Santos, 2012; Sandrím Julião; 2011; Rosevics, 2013; Maione de Souza, 2013; Gardini, 2010; Pereira y Assumpcao, 2014; Suppo, 2011).

La segunda característica, común a la mayoría de artículos, se compone de dos observaciones. Por un lado, se menciona a lo largo de los documentos que existen alternativas a la teoría, pero nunca las mencionan explícitamente (Romero, 2007; Vanegas, 2007; Sosa, 2006; Ballesteros, 2007; 2012;
Cid, 2008; Herrera, 2013). Por el otro, y como consecuencia de lo anterior, en lugar de propuestas concretas, las referencias se limitan al deber ser (Romero, 2007; Vanegas, 2007; Sosa, 2006; Ballesteros, 2007, 2012; Herrera, 2013; Murillo, 2013; Bacchi, 2013; Sandrím, 2011; Florencio, 2015; Elias, 2010).

La tercera característica es que la mayoría de autores entienden como sinónimo de pensamiento alternativo hacerle oposición a lo que se considera como Occidente (esto es, Europa y Estados Unidos). Además de las dificultades que entrańa considerar que América Latina no forma parte de Occidente, también es de señalar que no es posible ni suficiente tratar de avanzar teóricamente con el único fin de rechazar lo ya existente por su origen geográfico. Ideas en este sentido se encuentran en prácticamente todos los artículos estudiados, pero tienen mayor relevancia en los de Romero (2007), Vanegas (2007), Sosa (2006), Ballesteros (2007, 2012), Herrera (2013), de Oliveira y Geraldella (2013), Florencio (2015) y Ramos (2012).

Una cuarta característica que, además, pone en duda el carácter meramente latinoamericano de los aportes es que todos los autores construyen sus propuestas a partir de lo elaborado previamente por autores no latinoamericanos. De hecho, un sesgo importante en la literatura es que los autores utilizados para soportar las visiones propias son, en su mayoría, exponentes de visiones neomarxistas de las relaciones internacionales o de teorías críticas. Uno de los más utilizados es Immanuel Wallerstein, aunque también se encuentran Robert Cox, André Gunder Frank, Jacques Derrida, Antonio Gramsci, Michel 
Foucault, Ulrich Beck y el propio Karl Marx (Romero, 2007; Vanegas, 2007; Sosa, 2006; Ballesteros, 2007, 2012; Herrera, 2013; Faria, 2013; Ramos, 2012, 2013; Leme, 2013; Florencio, 2015; Akemi y Forigo, 2010; Scherma, 2012; Moll, 2012; Batista, 2012; Maione de Souza, 2014; Errejón, 2012; Zamudio y Arellano, 2011; Tello, 2010).

Resulta interesante, dentro de los autores mencionados, que, por un lado, se pretende criticar la visión occidental y, sin embargo, la mayoría de los que se utilizan son occidentales. Además, al haber tanto interés y concentración en los aportes hechos por Immanuel Wallerstein se debe resaltar que sus estudios tienen de referencia principalmente al continente africano. Así, ¿cómo se puede construir un pensamiento latinoamericano, supuestamente diferente de cualquier otro, si el soporte de las ideas refleja la realidad de otros contextos?

Una expresión de los problemas de esta forma de estructurar un pensamiento supuestamente independiente pero basado en conceptos construidos por autores externos a la región latinoamericana se encuentra en los aportes hechos por Ballesteros $(2007 ; 2012)$. Este autor parte de un concepto -posinternacional- que no fue creado por él sino por Rosenau (2000).

Desde el punto de vista de la observación de realidades diferentes a las exploradas por las teorías ya existentes de relaciones internacionales, la quinta característica común a los escritos analizados consiste en que, al contrario de lo que se podría pensar, muchos de los autores le dan prioridad al actor por excelencia del mainstream de las RI: el Estado. Esto no solo resulta de la importancia que, como se mencionó antes, se le da a los estudios de política exterior, sino que también se encuentra en los aportes teóricos que se hacen (Romero, 2007; Vanegas, 2007; Sosa, 2006; Ballesteros, 2007, 2012; Aguiar, 2011; Ramos, 2013; Sozzi, 2013; Leme, 2013; Ribeiro, 2014; Gaviao, 2015, Errejón, 2012). Lo anterior es importante debido a que si se pretende hacer un aporte diferenciado a lo ya existente, no es posible partir de la misma unidad de análisis (Del Arenal, 2007).

De hecho, autores que, como Herrera (2013), piden concentrarse en los movimientos sociales, las resistencias, el uso del lenguaje y otros aspectos, lo hacen desde una visión Estado-céntrica.

Una sexta característica consiste en dos observaciones relacionadas. Por una parte, existe una preocupación central en los aportes teóricos que tienen que ver con el concepto del desarrollo (Vanegas, 2007; Sosa, 2006; Ballesteros, 2007; Herrera, 2013; Ramos, 2013; Malamud, 2012; Castro Silva, 2008; Quintero, 2014; Fernández, 2014). Por otra parte, hay una visión -casi una obsesión- que nutre esta preocupación, por considerar que lo que se piensa, lo que se estudia, las estructuras sociales, políticas y económicas son resultado de una -aún no demostrada- dominación, imposición, subordinación o manipulación provenientes de eso que se denomina Occidente (Romero, 2007; Vanegas, 2007; Sosa, 2006, 2012; Herrera, 2013; De Oliveira y Geraldello, 2013; Ramos, 2012; Gallardo y Hassoun, 2014; Dallanguera, 2010).

De hecho, creer que lo existente es resultado de una intención de dominar / imponer por parte de las potencias se puede poner en 
entredicho a partir de una de las observaciones resultantes de este estudio. Como se mencionó, se encontró que aquellos países latinoamericanos que más estudios han publicado sobre teoría de relaciones internacionales en términos proporcionales son las consideradas potencias regionales. De esta manera, si se tiene en cuenta que los principales productores de conocimiento en esta disciplina son, en su orden, Estados Unidos y Europa, podría pensarse que su carácter de potencias globales y abiertas (el contexto) determina la producción de conocimiento y no al contrario. Esto es, ser potencias con interés de influir en el ámbito internacional desencadena procesos -aún por estudiar- que reflejan la necesidad de comprender el mundo.

Si persiste la visión contraria -es decir, que por la manipulación y dominación se consolidan como potencias-, tendría que preguntarse si la producción intelectual, aún creciente, de países emergentes como Brasil o México significa que estos imponen $s u$ visión latinoamericana al resto de América Latina.

Esto último, así como algunas de las características mencionadas, configuran una séptima característica consistente en la contradicción de algunos de los postulados que pretenden ser aportes a la teoría. Por ejemplo, para Romero (2007, p. 149), el conocimiento, la metodología y los aportes de países como Japón, Corea o China son deliberadamente excluidos de la visión occidental. No obstante, a renglón seguido afirma que esos conocimientos, metodologías y aportes permitieron el desarrollo de la ciencia europea y estadounidense. Otro ejemplo se da en el texto de Vanegas (2007). Para la autora, el concepto de ideología se ha marginado -también deliberadamente- de los estudios internacionales. Sin embargo, su texto demuestra todo lo contrario. Un último ejemplo se observa en el escrito de Sosa (2006), quien describe lo que denomina como la "época dorada" del pensamiento latinoamericano pero que incluye teorías foráneas como la de la modernización ${ }^{3}$ y del sistema mundo ${ }^{4}$.

Aparte de las características mencionadas, el escrito de Sosa (2006) demuestra la falta de producción teórica desde la segunda mitad del siglo xx en América Latina. De hecho, podría pensarse que la supuesta necesidad de contar con un pensamiento latinoamericano se debe más a la ańoranza por ese pasado que, como Sosa, algunos consideran la "época dorada" del pensamiento regional.

Desde el punto de vista teórico y científico, todos los artículos analizados tienen un problema de fondo: parten de supuestos y asumen parámetros, variables o conceptos que se caracterizan por su falta de claridad, definición, delimitación y, por tanto, de la posibilidad de su demostración, no solo empírica, sino a partir del pensamiento argumentativo. Algunos autores hablan de una crisis de las relaciones sociales, pero no la definen. Otros hablan de la crisis de la modernidad y no la demuestran. Otros más afirman la existencia

Iniciada por Walt W. Rostow.

Propuesta por Immanuel Wallerstein. 
de un mainstream en el pensamiento de las relaciones internacionales pero todos utilizan autores que ellos mismos afirman han sido excluidos o parten de metodologías que ese mainstream propone.

A partir de las anteriores observaciones, se puede plantear qué tan originales son las propuestas latinoamericanas acá incluidas, a partir de los criterios establecidos al principio de este apartado (ver anexo 2).

Así las cosas, ninguno de los artículos analizados puede considerarse como original en los términos acá establecidos, puesto que ninguno cumplió más de un criterio. De esta manera, a partir de los análisis cuantitativo y cualitativo se puede evidenciar que no hay un pensamiento propio latinoamericano en RI. No obstante, persiste la duda de si realmente esto es necesario.

\section{LA NO NECESIDAD DE UNA TEORÍA LATINOAMERICANA}

No se puede hablar de un pensamiento latinoamericano en relaciones internacionales, por lo menos entre 2006 y 2014, en las publicaciones analizadas. Pero construirlo se considera, como se señaló en la introducción, una necesidad, entendida esta como utilidad para la explicación de la realidad internacional desde un punto de vista latinoamericano. La pregunta que persiste es, ¿para qué? Este apartado pretende contribuir al debate defendiendo la visión contraria: no existe un pensamiento latinoamericano porque no es necesario.

Dentro de las razones principales para demostrar la anterior aseveración encontramos las siguientes, aunque no por eso las únicas.
Primero, porque las teorías son modelos para interpretar la realidad y esos modelos no dependen de la nacionalidad del autor. No importa que este sea del sur o de un país pobre o de uno rico, lo que importa es qué tan válida es la propuesta de interpretación en relación con las demás disponibles y con lo que se percibe de esa realidad. Por ejemplo, el realismo fue válido en su momento para explicar una situación específica, no por el origen de sus principales exponentes sino por su universalidad de principios.

De hecho, si el determinante fuera el origen nacional del autor, tendría que demostrarse que, por ser alemán, Hans Morgenthau llegó a las conclusiones que llegó y, además, cómo este origen permitió que sus aportes fueran generalizados de la manera que lo fueron. Si el determinante hubiera sido su origen alemán, ¿cómo explicar que, en el momento en que Morgenthau pensaba en lo que hoy conocemos como realismo, Alemania estaba inmersa en una lógica de superación de las fronteras nacionales y del interés nacional en favor de la integración europea?

Segundo, porque la realidad latinoamericana no es diferente de la realidad en las demás regiones del mundo. Es cierto, los problemas pueden ser distintos, así como las necesidades $y$, por tanto, las inquietudes. No obstante, los actores en el ámbito internacional de la región no son "naturalmente" diferentes a los países de Europa, de África o de Asia, incluso, de Estados Unidos. En consecuencia, las lógicas de actuación pueden ser explicadas a través de las teorías ya existentes. No sería, entonces, necesaria una visión propia latinoamericana que, además, resultaría excluyente y, por 
tanto, de difícil generalización. Si el objetivo es construir ciencia a partir de la teoría, esta siempre debe ser incluyente.

Un caso interesante es la observación de realidades que no han sido exploradas por otras teorías o que no han sido tenidas en cuenta. Sin embargo, como demostró el análisis de los artículos en este estudio, esas otras realidades tampoco han sido identificadas o abordadas por los autores latinoamericanos, por lo menos en las publicaciones revisadas.

Tercero, porque las fuentes de interpretación de la realidad -incluidas las provenientes de las teorías críticas o marxistas- están basadas en el proceso de investigación científico, en las epistemologías y las ontologías que se originaron en Occidente. De hecho, a través de futuras investigaciones se podría evidenciar que esta observación es válida para cualquier parte del mundo. No es una cuestión de imposición sino que es en la civilización occidental, como lo demuestran autores como Ferguson (2012), donde más se desarrolló el método científico, único capaz de generar teorías. Las causas no son de superioridad, sino del desarrollo de esas sociedades y también de la casualidad.

Cuarto, porque no ha habido, como se mostró, un interés genuino por reflexionar sobre las teorías de las relaciones internacionales en la región. Esto se puede deber a que la realidad latinoamericana muestra un proceso de construcción vigente del Estado. Esto llevaría a que la concentración principal de publica- ciones se encuentre en la ciencia política y en la discusión del tema del desarrollo ${ }^{5}$. En este sentido, a diferencia de los países europeos y de Estados Unidos, en donde la estabilidad de la organización estatal permitió concentrarse en la interacción de múltiples actores en el ámbito más allá de las fronteras, y en explicar las dinámicas de esas interacciones, en América Latina, la situación constantemente cambiante de los gobiernos, de los modelos que estos implementan, de su relación con la sociedad y con los demás países lleva a que la reflexión teórica internacional pase a un segundo plano. De esta manera, podría sostenerse que tal vez en el futuro habrá unos mayores aportes teóricos sin que esto quiera decir que vayan a ser novedosos o diferentes a los ya existentes.

Muchas otras razones podrían esgrimirse para sustentar esta posición. No se trata de considerar que la señalada acá sea la verdad absoluta en términos de la deseabilidad o no de una teoría "latinoamericana". Sin embargo, este debate debe darse puesto que tiene implicaciones en la relevancia y rigurosidad de la disciplina en el plano regional. La necesidad se debe demostrar y, para ello, es necesario comenzar por un debate académico sobre el tema y no darla como un hecho indiscutible, absoluto; visión que tanto rechazan las perspectivas críticas y pospositivistas de las relaciones internacionales. No se trata de afirmar que los latinoamericanos no tienen nada qué decir o aportar a la teoría de las relaciones interna-

\footnotetext{
5 Aunque no se tomaron en cuenta los demás artículos que no fueran de TRI, la observación general es que las publicaciones consideradas de relaciones internacionales se limitan a hacer descripciones acerca de políticas exteriores de los países, de discusión en torno al impacto del capitalismo y la globalización, así como de movimientos sociales.
} 
cionales. Se trata de cuestionar si lo mucho que hay para hacer depende exclusivamente de las características culturales, sociales o de nacionalidad de los académicos de esta región.

\section{CONCLUSIÓN}

Existe una-casi-obsesión en América Latina por ser diferentes. Se critican los modelos económicos simplemente por ser "importados"; se critica la falta de una identidad latinoamericana; se espera la construcción de un modelo político latinoamericano. Esta expectativa de crear algo diferente, solo por el hecho de ser latinoamericanos, también ha permeado el ámbito académico, incluido el de los estudios internacionales.

No obstante, como se planteó al inicio de este artículo, una teoría propia desde un punto de vista regional ni existe ni es necesaria.

A esta conclusión se puede llegar después de examinar los escritos que, en diferentes países, se publican sobre reflexión teórica. Lo primero que llama la atención es que casi no hay reflexión. Lo segundo es que la poca que hay no es innovadora sino que se limita a revisar o aplicar las teorías ya existentes.

Este estudio no es suficiente, sin embargo, para afirmar de manera contundente lo anterior. Es una aproximación inicial a una investigación sobre lo que se publica acerca de temas internacionales en la región. No fueron incluidas todas las publicaciones, ni todos los autores ni todos los países. Es tan solo una muestra que podrá tener desarrollos futuros. De igual manera, es cierto que la visión acá planteada podría considerarse como positivista en su visión de la construcción del conocimiento. No obstante, como se señaló, aunque se mencione la posibilidad de la existencia de alternativas al positivismo, estas no han sido explícitas hasta el momento.

Pero, además, como se mencionó en el apartado correspondiente, tampoco parece ser necesaria. Las teorías sirven para explicar la realidad. Son modelos, son imperfectas, son limitadas. Pero es así porque el ser humano, en su limitación mental, no puede comprender de manera absoluta la realidad que lo rodea.

Lo anterior no es necesariamente una mala noticia. Lo preocupante es que no haya suficiente reflexión teórica. No obstante, el que no sea necesario contar con un pensamiento diferente a lo ya existente, puede permitir aprovechar lo ya creado y contribuir al conocimiento de la humanidad y no solo al latinoamericano. Una visión desde lo latinoamericano o desde el Sur no es sino una forma excluyente, incluso xenófoba, de pensar el mundo, que es, en últimas, lo que se critica en América Latina. Por su parte, es necesario que se supere la preeminencia que han tenido los temas de desarrollo sobre la agenda de investigación de disciplinas que son más amplias, como las relaciones internacionales.

Además, las más de las veces, la búsqueda de una supuesta vía latinoamericana lo que hace es profundizar la creencia y adopción de pensamientos, ideologías o modelos basados, no en pensamientos autóctonos, pero sí en un rechazo a los valores y principios de las civilizaciones que han tenido no solo éxito económico, político y social, sino también que han contribuido al avance científico en el mundo. Adoptar los aportes hechos desde el marxismo y sus múltiples expresiones en las 
teorías de las relaciones internacionales, no por representar resistencias a las realidades de la sociedad occidental, dejan de ser occidentales.

Por último, ¿qué es América Latina sino también una creación artificial a partir de intereses políticos? De allí la dificultad para establecer y delimitar lo que se puede entender por latinoamericano y, claro está, sus expresiones académicas y teóricas. El debate no está en demostrar la existencia de la región o de su carácter diferenciado, sino más bien en reconocer que la ciencia puede y debe seguir avanzando pero esto no se hace a través de líneas étnicas, culturales o nacionales, ni rechazando lo ya existente.

\section{REFERENCIAS}

Aguiar Cunha, D. M. (2011). Estabilidade e os Meios para Alcançá-la: Uma Abordagem Neo-realista. Caderno de Relaçōes Internacionais, 2 (3), 67-72.

Akemi Sawasaki, C. y Vinicius Forigo, M. (2010). Uma análise da guerra no iraque com base nas ideias de Michel Foucault e Joseph Nye. Revista Relaçôes Internacionais no Mundo Atual, 2 (12), 10-46.

Alves Ribeiro, M. M. L. (2013). A Escola Inglesa das Relaçôes Internacionais como Instrumental Teórica Adequado de Ánalise das Intervençóes Humanitárias. Examãpaku, 6 (2).

Anzaldi, P. A. (2008). Raymond Aron y la teoría de las relaciones internacionales. Revista de Estudios Internacionales, (159), 9-32.

Anzaldi, P. (2009). Clausewitz y la teoría de las relaciones internacionales. Revista de Estudios Internacionales, (164), 31-54.

Bacchi Hora, G. (2013). Tobias Barreto e a Crítica Moderada Ao Positivismo. Caderno de Relaçōes Internacionais, 4 (7), 97-121.
Ballesteros Pérez, C. (2007). Tesis para la reconstrucción de la teoría postinternacional. Revista de Relaciones Internacionales de la UNAM, (99), 27-39.

Ballesteros Pérez, C. (2012). Sociedad, naturaleza, culturas. Contribución a un pensamiento posinternacional. Revista de Relaciones Internacionales de la UNAM, (112), 67-99.

Batista Leite, L. A. (2012). O discurso como objeto de estudo e instrumento metodológico nas Relaçóes Internacionais. Monçôes: Revista de Relaçōes Internacionais da UFGD, Dourados, 1 (1).

Becerra, L. (2013). Hacia un marco constructivista realista para el análisis de las relaciones internacionales desde las periferias. Revista Colombia Internacional, (78), 79-126.

Bernal Meza, R. (2013). Heterodox Autonomy Doctrine: Realism and Purposes, and its Relevance. Revista Brasileira de Politica Internacional, 56 (2), 45-62.

Castro Silva, G. J. y Olivieri, A. G. (2006). Revisando a Teoria das Relaçóes Internacionais. Enfoque Imperial: O Surgimento de Uma Nova Teoria? Hegemonia, (1).

Castro Silva, G. J. (2008). A Teoria da Dependência: Reflexôes Sobre Uma Teoria Latino-Americana. Hegemonia, (3).

Castro, T. (2013). Debates da Conjuntura Internacional Pós-Bipolaridade: O Realismo Ofensivo (Pessimista) de Mearsheimer e o Realismo de Choque Civilizatório de Huntington e Suas Repercussôes. Caderno de Relaçôes Internacionais, 4 (7), 133-148.

Carvajal H. L. (2009). Posmodernismo y constructivismo: su utilidad para analizar la política exterior colombiana. OASIS (14), 201-218.

Cervo Amado, L. (2008). Conceitos em Relaçóes Internacionais. Revista Brasileira de Politica Internacional, 51 (2), 8-25. 
Cid Capetillo, I. (2008). Avances y aportaciones sobre teoría de relaciones internacionales. Revista de Relaciones Internacionales de la UNAM, (100), 33-50.

Creus, N. (2013). Las falacias del realismo periférico. Un análisis en torno a sus costos como estrategia de política exterior. Relaciones Internacionales (44).

Dallanguera Pedraza, L. (2010). Teoría y metodología de la geopolítica. Hacia una geopolítica de la “construcción de poder". Revista Mexicana de Ciencias Politicas y Sociales, 52 (210).

Dallanegra Pedraza, L. (2009). Toma de decisiones y construcción de poder desde el realismo sistémico estructural. Revista de Relaciones Internacionales de la UNAM (104), 11-50.

Das Graças Pinto, J. H. (2013). A Influência de Mancur Olson sobre a Teoria da Estabilidade Hegemônica de Robert Gilpin. Conjuntura Global, 2 (3), 165-172.

De Melo Santos, A. (2012). O Realismo Na Teoria das Relaçôes Internacionais. Caderno de Relaçóes Internacionais, 3 (5), 84-92.

De Sá Gurmarães, F. (2008). O Debate entre Comunitaristas e Cosmopolitas e as Teorias de Relaçóes Internacionais: Rawls Como Uma Via Média. Contexto Internacional, 30 (3), 571-614.

Del Arenal, C. (2007). Introducción a las relaciones internacionales. Madrid: Tecnos.

Dutra, L. (2015). A escola inglesa das relaçóes internacionais e a perspectiva histórica do espaço internacional. Revista Relações Internacionais no Mundo Atual, 1 (20), 72-95.

Elias Riche, F. (2010). Paradigmas, Teorias e Conceptos em Relacoes Internacionais: uma análise metateórica. Século XXI, 1 (1).

Errejon Galvan, I. (2012). ¿Qué es el análisis político? Una respuesta desda la teoría del discurso y la hegemonía. Relacso, 1-16.

Escudé, C. (1991). La política exterior de Menem y su sustento teórico implícito. América Latina Internacional, 8 (27).

Escudé, C. (1992). Realismo periférico. Argentina: Planeta.

Escudé, C. (1995). El realismo de los Estados débiles. Argentina: GEL/NuevoHacer.

Estrella Faria, L. A. (2013). The Value of the Concept of Hegemony for International Relations. Austral: Brazilian Journal of Strategy and International Relations, 2 (3), 193-216.

F. de Oliveira, M. y S. Geraldella, C. (2013). Maquiavel, os Realistas e a Politica Internacional. Brazilian Journal of International Relations, 2 (1), 160-177. Ferguson, N. (2012). Civilización: Occidente y el resto. España: Debate.

Fernandes Maso, T. y Rodrigues Sélis, L. M. (2012). $\mathrm{Na}$ véspera do tempo: repensando as Relaçóes Internacionais. Monçōes: Revista de Relaçôes Internacionais da UFGD, 1 (1).

Fernández-Guillén, O. E. (2014). Celso Furtado: visión y vigencia del desarrollo y la integración en América Latina. Cuadernos sobre Relaciones Internacionales, Regionalismo y Desarrollo, 9 (18), 87-134. Florencio, F. (2015). Pós-Estruturalismo e Neorrealismo: Críticas e Perspectivas nas Relaçóes Internacionais. Conjuntura Global, 4 (2), 262-273.

Gallardo Guillén, L. C. y Hassoun Hassoun, E. (2014). El dependentismo, el desarrollo y la integración regional en América Latina. Cuadernos sobre Relaciones Internacionales, Regionalismo y Desarrollo, 9 (18), 169-200.

Gardini, G. L. (2010). Who still cares about the English School, and why? Mural Internacional, 1 (1).

Gaviao, L. (2015). Identidades supranacionais, Mural Internacional, 6 (1).

Griffiths, M., Roach, S. C. y Solomon, M. S. (2008). Fifty Key Thinkers in International Relations. EE.UU.: Routledge Key Guides. 
Guzzini, S. (2015). Benjamin Cohen on Global Political Orders: When Keynes Meets Realism - and Beyond. Contexto Internacional, 37 (3), 851-887.

Herrera Santana, D. (2013). La teoría, las relaciones internacionales y las grandes transformaciones mundiales en el siglo XXI. Apuntes para repensar el mundo y sus interpretaciones. Revista de Relaciones Internacionales de la UNAM, (117), 11-37.

Leme Barbosa, T. A. (2013). O Conceito de Estado em Immanuel Wallerstein e Hans Morgenthau: Alguns Apontamentos Teóricos. Conjuntura Global, 2 (1), 29-33.

Lozurdo, D. (2013). Fichte, A Revoluçáo Francesa e o Ideal da Paz Perpétua. Brazilian Journal of International Relations, 2 (1), 179-231.

Maione de Souza, E. (2008). Re-evaluating the Contribution and Legacy of Hedley Bull. Brazilian Political Science Review, 3 (1), 96-124.

Maione de Souza, E. (2013). A Escola Inglesa de Relaçōes Internacionais e o Direito Internacional. Mural Internacional, IV (1).

Maione De Souza, E. (2014). Justiça de transição na teoria das Relaçôes Internacionais: realismo, construtivismo e as possibilidades de um engajamento crítico. Monçôes: Revista de Relaçôes Internacionais da UFGD, 3 (6).

Malamud, A. (2012). Conceptos, teorías y debates sobre la integración regional. Brazilian Journal of International Relations, 1 (3), 367-397.

Moll, R. (2012). Gramsci e as Relaçoes Internacionais: para superar a reificacao do estado e a anarcofilia. Revista Monçōes, 1 (1).

Murillo Zamora, C. (2013). Relaciones internacionales: algunas consideraciones disciplinarias y teóricas. Revista de Relaciones Internacionales de la UNAM, (116), 11-41.

Pablo Tello, Á. (2010). La teoría de las relaciones internacionales desde un punto de vista político- polemológico. Sistema mundo y uso de la fuerza: nuevos escenarios y actores. El rol del instrumento militar y los caminos hacia la paz. Relaciones Internacionales (39).

Papini, R. (2009). Para una teoría maritainiana de las relaciones internacionales. Revista de Estudios Internacionales, (163), 47-61.

Pedroso Mendes, F. (2012). Clausewitz, o Realismo Estrutural e a Paz Democrática: Uma Abordagem Crítica. Contexto Internacional, 34 (1), 79-111.

Pereira, D. C. y Assumpcao Rocha, R. (2014). Debates teóricos em Relacoes Internacionais: origen, evolucao e perspectiva do embate Neo-Neo. Revista de Relaçōes Internacionais da UFGD, 3 (6).

Pereira Rezende, L. (2014). Teoria Realista Ofensiva de Cooperação em Defensa na Unipolaridade. Contexto Internacional, 36 (2), 519-548.

Prada Álvarez, N. E. y Quintero Rizzuto, M. L. (2014). Contribuciones de Aníbal Pinto en el marco del estructuralismo latinoamericano: desarrollo e integración regional. Cuadernos sobre Relaciones Internacionales, Regionalismo y Desarrollo, 9 (18), 135-168.

Quintero Rizzuto, M. L. (2014). El estructuralismo latinoamericano bajo la égida de Prebisch y la CEPAL: desarrollo e integración regional. Cuadernos sobre Relaciones Internacionales, Regionalismo y Desarrollo, 9 (18), 43-85.

Ramos, L. (2012). Ordem e Poder na Economia Política Global: A Contribuição Neogramsciana. Contexto Internacional, 34 (1), 113-150.

Ramos, L. (2013). Critically Thinking the Global Political Economy: Assessments for the Study of Middle Emerging Powers. Austral: Brazilian Journal of Strategy and International Relations, 2 (3), 217-239.

Ribeiro Barao, G. (2014). Cultura e diplomacia cultural no século XxI: roposta de revisão do pensamento 
brasileiro de relaçóes internacionais. Monçōes: Revista de Relaçôes Internacionais da UFGD, 3 (5).

Romero Castilla, A. (2007). Una perspectiva no eurocéntrica para el estudio de las relaciones internacionales: el este de Asia. Revista de Relaciones Internacionales de la UNAM, (99), 143-157.

Rodrigues, N. (2014). Teoria da Interdependência: Os Conceitos de Sensibilidade e Vulnerabilidade nas Organizaçôes Internacionais. Conjuntura Global, 3 (2), 107-116.

Rosenau, J. (2000). Beyond Postinternationalism. En Hobbs, H. H. Pondering Postinternationalism. A Paradigm for the Twenty First Century? Estados Unidos: State University of New York.

Rosevics, L. (2013). Contribuicoes da École des Annales e de Fernand Braudel para as Relacoes Internacionais. Relacoes Internacionais do Mondo Actual, 1 (17).

Russell, R. (1991). El 'neoidealismo periférico': un esquema para orientar la política exterior de los países del Cono Sur en la posguerra fría. América Latina Internacional, 8 (29).

Salatini, R. (2013). O Tema da Paz Perpétua. Brazilian Journal of International Relations, 2 (1), 141-158.

Sánchez, L. E. (2012). ¿¿De qué se habla cuando se habla de constructivismo? Revista de Relaciones Internacionales de la UNAM, (114), 107-129.

Sandrím Julião, T. (2011). Teoría e História das Relações Internacionais: Uma Escola Latino-Americana? Conjuntura Austral, 2 (7), 51-66.

Scherma, M. A. (2012). As fronteiras nas Relaçóes Internacionais. Monçöes: Revista de Relaçōes Internacionais da UFGD, 1 (1).

Silva Costa, C. Â. y Britto dos Santos, L. (2013). Uma Abordagem da Teoria do Complexo de Segurança sobre as águas transfronteiriças do Rio Jordáo. Mural Internacional, IV (1).
Sombra Saraiva, J. M. (2006). Revisitando a Escola Inglesa. Revista Brasileira de Politica Internacional, 49 (1), 131-138.

Sosa Fuentes, S. (2006). Modernización, dependencia y sistema mundo: los paradigmas del desarrollo latinoamericano y los desafíos del siglo xxI. Revista de Relaciones Internacionales de la UNAM, (96), 88-121.

Sosa Fuentes, S. (2012). Otro mundo es posible: crítica del pensamiento neoliberal y su visión universalista y lineal de las relaciones internacionales y el sistema mundial. Revista Mexicana de Ciencias Politicas y Sociales, 54 (214).

Sozzi Miguel, F. M. (2013). Threat Perception in International Relations: The Realist and the Liberal Accounts. Brazilian Journal of International Relations, 2 (3), 484-509.

Suppo, H. R. (2011). A importancia do chamado soft power no paradigma realista clásico. Mural Internacional, 2 (2).

Tickner, A. y Wæver, O. (eds.) (2009). International Scholarship Around the World. Estados Unidos: Routledge.

Tickner, A. y Blaney, D. L. (eds.) (2013). Claiming the International. Worlding Beyond the West. Estados Unidos: Routledge.

Treinto, M. (2008). O Tema da Guerra na Escola Inglesa das Relaçōes Internacionais. Contexto Internacional, 30 (1), 171-208.

Vanegas Vargas, R. (2007). La ideologización de la ideología: los desafíos para el estudio de las relaciones internacionales. Revista de Relaciones Internacionales de la UNAM, (99), 173-186.

Zamudio González, L. y Arellano Gault, D. (2011). Más allá de las patologías de la burocracia: introduciendo la teoría de las organizaciones al estudio de las or. Revista Mexicana de Ciencias Políticas y Sociales, 53 (213). 
ANEXO 1

\section{Argentina}

- Relaciones Internacionales - La Plata

- RESGa (no disponible en Internet)

- Revista Argentina de Relaciones Internacionales

- Revista Colección

- Revista de Economía y comercio internacional (no corresponde a la temática)

- Revista Facultad de C.C. Económicas (no corresponde a la temática)

- Revista del CeId (artículos de análisis y opinión)

- Series: estudios e investigaciones (no disponible en Internet)

- Serie notas del CEI (no disponible en Internet)

- Sociedad Global (no disponible en Internet)

- Anuario en Relaciones Internacionales (no disponible en Internet)

- Argentina Global (no disponible en Internet)

- Colección Programas de investigación en Relaciones Internacionales (no disponible en Internet)

- Contexto internacional

- Cuadernos de política exterior argentina (no es revista, son working papers)

- De sur a norte (no disponible en Internet)

- En Letra (no disponible en Internet)

- Estrategia (no disponible en Internet)

- Agora Internacional (no disponible en Internet)

- $\quad$ IEsPyc (no disponible en Internet)
- Informe de coyuntura del IERAL (no es una revista)

- Journal de Ciencias Sociales (no disponible en Internet)

- Libra (no disponible en Internet)

\section{CHILE}

- Estudios internacionales

- Carta cronológica de las relaciones internacionales chilenas (no disponible en Internet)

- Cono sur (no disponible en Internet)

- Estudios transfronterizos (no disponible en Internet)

- Latinamerican Journal of International Affairs (pocos números no representativos)

- Revista chilena de cooperación (no disponible en Internet)

- Revista digital Parzinas (no disponible en Internet)

- Revista focus eurolatino (no disponible en Internet)

- Revista Fuerzas armadas y sociedad (solo del 1985 a 1988)

- Tratados de Chile (no corresponde a artículos científicos)

\section{MÉXICO}

- Confines

- Revista de Relaciones Internacionales

- Foro internacional

- Anuario mexicano de Relaciones Internacionales (solo disponibles algunos elementos, ninguno de teoría) 
- Partes: Revista Mexicana de estudios sobre la Cuenca del Pacífico (no corresponde al tema)

- CIMEXUS

- Cuadernos del Centre de Relaciones Internacionales (no disponible en internet)

- Cuadernos de política exterior mexicana (no disponible en Internet)

- Cuadernos de política internacional (oficial, no académica)

- Cuadernos Imred (no disponible en internet)

- Estudios del tercer mundo (no disponible en Internet)

- $\quad$ Foreign affairs en español (no disponible en Internet)

- Foreign affairs Latinoamerica (no académica)

- México y la Cuenca del Pacífico

- Revista Norteamericana

- Relasco

- Revista mexicana de cc Políticas y Sociología

- Migraciones internacionales (no corresponde a la temática)

- Portes (no disponible en Internet)

- Retos internacionales (no disponible en Internet)

- Revista intercontinental "Ducit et Docet” de investigación (no disponible en Internet)

- Revista mexicana de estudios canadienses (no corresponde a la temática)

- Revista mexicana de política exterior (no disponible en Internet)

- Tendencias (no disponible en Internet)

- Urbi et Orbi (no disponible en Internet)

\section{BRASIL}

- World Citizen magazine (no suficientes números)

- Via mundi (no disponible en Internet)

- Universitas Relacoes Internacionais (no disponible en internet)

- Tensoes mundiais

- Seculo XXI

- Revista Neiba: Cadernos Argentina-Brasil (no corresponde a la temática)

- Revista de Sociología y Política (no corresponde a la temática)

- Revista de Iniciaçao científica en Relaçoes Internacionais (solo un año disponible)

- Revista de historia Bilros (no corresponde a la temática)

- Revista de estudios sociais (no corresponde a la temática)

- Revista de estudios internacionais (no disponible en internet)

- Revista da escola de guerra naval (no corresponde a la temática)

- Revista brasileira de política internacional

- Relaçoes internacionais domondo actual

- O cosmopolitico

- Network (no disponible en Internet)

- Mural internacional

- Moncoes

- Meridiano 47 (no disponible en Internet)

- Contexto internacional

- Estudios internacionais (disponibles pocos números)

- Examapaku

- Fronteira (solo disponible hasta 2011)

- Hegemonia

- Intellector (no disponible en Internet)

- Leviathan (diponibles pocos números) 
- Revista Brasileira

- Austral: Revista brasileira de estrategia y Relacoes Internacionais

- Brazilian journal of International Relations

- Cuaderno de Relacoes Internacionais

- Brazilian journal of Latinamerican studies (no disponible en internet)

- Cena internacional (pocos números disponibles) ( $\mathrm{n}^{\circ} 7$ Brasil)

- Coyuntura austral

- Brazilian Political Science Review

- Coyuntura global

\section{Venezuela}

- Cuadernos sobre Relaciones internacionales, regionalismo y desarrollo

- Perfiles internacionales (no disponible en Internet)

- Perfil internacional (no disponible en Internet)

- Revista geográfica venezolana (pocos números disponibles)

\section{Colombia}

- OASIS

- Colombia internacional

- Perspectivas internacionales (no disponible en Internet)

- Revista de relaciones internacionales, estrategia y seguridad (no disponible en Internet)

- Vox populi (no disponible en Internet)

- Folios (no disponible en Internet)

\section{Perú}

- Agenda internacional

\section{ECUADOR}

- Ciencias políticas y relaciones internacionales (solo un número disponible)

- Iconos

- Valor agregado (no disponible en Internet)

- Revista empresarial (no corresponde a la temática)

- Revista Sarauce (no disponible en Internet)

- Revista de estudios de seguridad (no corresponde a la temática)

\section{URUGUAY}

- Revista del sur (pocos números disponibles)

\section{BoliviA}

- Revista diplomática e internacional (no disponible en Internet)

\section{República Dominicana}

- Revista dominicana de política exterior (no disponible en Internet)

\section{CuBA}

- Tricontinental (no disponible en Internet) 


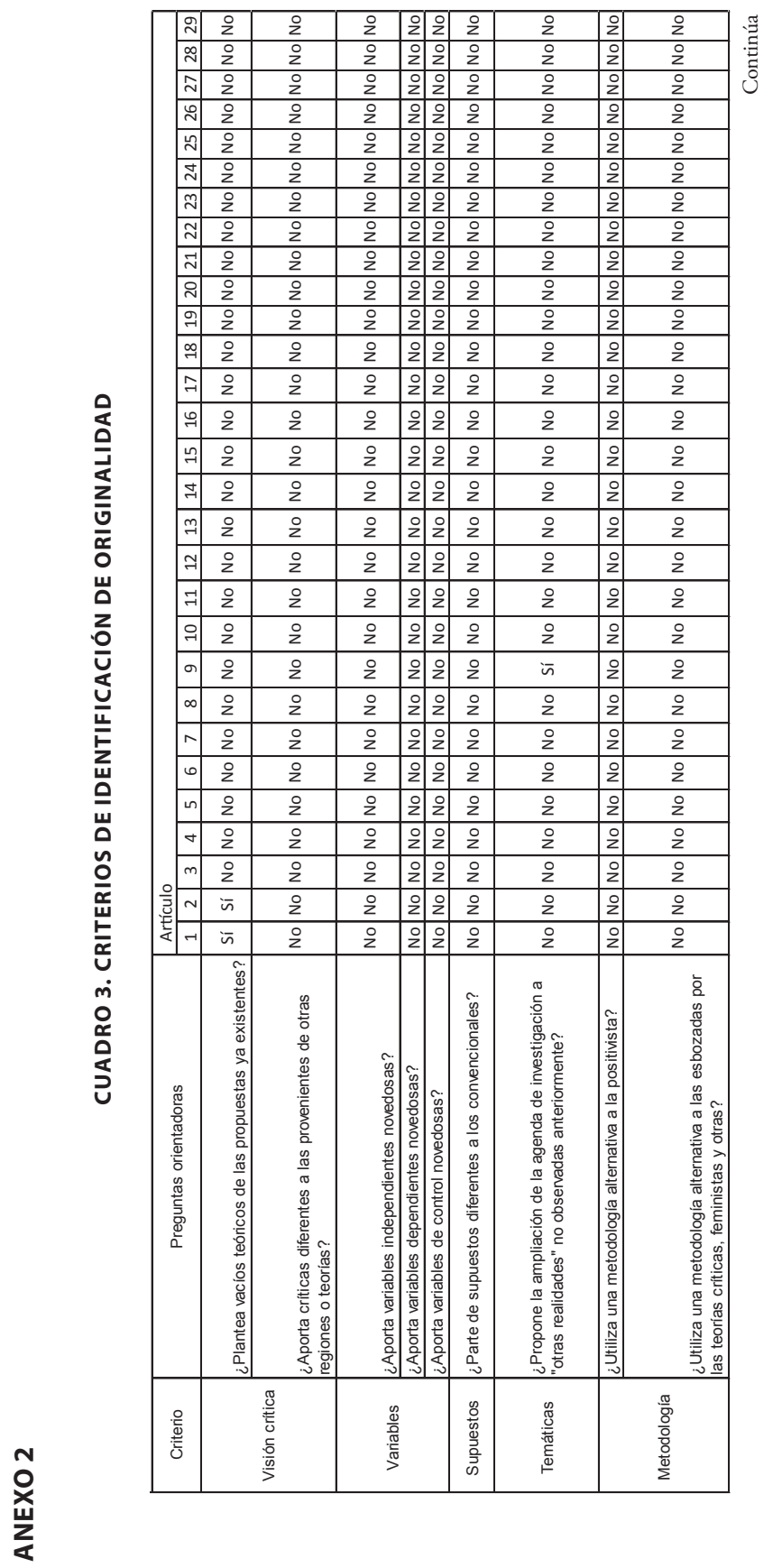




\begin{tabular}{|c|c|c|c|c|c|c|c|c|}
\hline . & $\frac{o}{2}$ & $\frac{\circ}{2}$ & $\frac{0}{2}$ & $2:$ & 알 & zo & 2 & 울 \\
\hline \begin{tabular}{|l|} 
fo \\
\end{tabular} & z & ㅇ & $\stackrel{\circ}{z}$ & $2 \frac{1}{2}$ & z & $\frac{o}{z}$ & 2 & $\frac{o}{z}$ \\
\hline (6) & $\bar{n}$ & $\frac{0}{2}$ & $\frac{1}{2}$ & 20 & $\frac{0}{2}$ & 운 & 20 & 울 \\
\hline \begin{tabular}{|l|}
6 \\
\end{tabular} & $\frac{o}{z}$ & $\stackrel{\circ}{z}$ & $\frac{0}{2}$ & ¿20 & $\frac{1}{2}$ & $\frac{1}{2}$ & $\frac{10}{2}$ & $\frac{1}{2}$ \\
\hline | & $\frac{o}{z}$ & $\stackrel{\circ}{z}$ & $\frac{\circ}{2}$ & ¿: & $\frac{1}{2}$ & $\frac{1}{2}$ & 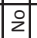 & $\frac{1}{2}$ \\
\hline \begin{tabular}{|l|l}
8 \\
\end{tabular} & z & 을 & $z_{2}^{\circ}$ & $\begin{array}{lll} & 2 & 0 \\
\end{array}$ & z & $\frac{0}{2}$ & 2 & $\frac{0}{z}$ \\
\hline की & $\frac{0}{z}$ & $\frac{0}{z}$ & 을 & $\begin{array}{ll} & 0 \\
2\end{array}$ & \& & z & \begin{tabular}{|l|}
2 \\
\end{tabular} & z \\
\hline 市 & i & 울 & $\frac{\circ}{2}$ & $2 \%$ & $\frac{0}{2}$ & $\frac{0}{2}$ & 2 & $\frac{0}{2}$ \\
\hline in & ㅇ & 을 & $\stackrel{\circ}{2}$ & ¿20 & $\frac{\circ}{z}$ & z & 2 & z \\
\hline \begin{tabular}{|l|} 
in \\
\end{tabular} & $\stackrel{\circ}{z}$ & 을 & $\stackrel{\circ}{2}$ & $2 \frac{10}{2}$ & $\frac{o}{z}$ & $\frac{0}{2}$ & $\begin{array}{ll} \\
2\end{array}$ & $\frac{o}{z}$ \\
\hline 包 & $\bar{n}$ & $\frac{\circ}{2}$ & $\frac{0}{2}$ & $\begin{array}{lll}10 & 2 \\
\end{array}$ & $\frac{o}{z}$ & $\frac{1}{2}$ & 20 & $\frac{0}{2}$ \\
\hline \begin{tabular}{|l|} 
f \\
\end{tabular} & i & 을 & $\frac{\circ}{2}$ & ¿2: & $\frac{o}{z}$ & $\frac{0}{2}$ & 2 & $\frac{0}{2}$ \\
\hline \begin{tabular}{|l|} 
m \\
\end{tabular} & $\bar{n}$ & 을 & $\stackrel{\circ}{2}$ & $2 \frac{10}{2}$ & : & $\frac{0}{2}$ & \begin{tabular}{l|}
2 \\
\end{tabular} & $\frac{o}{z}$ \\
\hline 중 & $\stackrel{\circ}{z}$ & $\stackrel{\circ}{z}$ & $\stackrel{\circ}{2}$ & 22 & $\frac{o}{z}$ & $\frac{1}{2}$ & 잉 & $\frac{0}{2}$ \\
\hline ही & 을 & 울 & $\stackrel{\circ}{z}$ & ¿2: & $\frac{o}{z}$ & $\frac{0}{2}$ & 2 & $\frac{0}{2}$ \\
\hline \begin{tabular}{|l|} 
in \\
\end{tabular} & zo & 인 & $\frac{0}{2}$ & \begin{tabular}{l|l|l}
2 & 0 \\
\end{tabular} & \begin{tabular}{|l}
$\circ$ \\
2
\end{tabular} & zo & 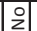 & $\frac{1}{2}$ \\
\hline g & $\bar{n}$ & $\frac{0}{2}$ & $\frac{0}{z}$ & $\begin{array}{lll}1 & 0 \\
2 & 0\end{array}$ & $\frac{0}{z}$ & $\frac{0}{2}$ & \begin{tabular}{|l|} 
\\
2
\end{tabular} & z \\
\hline \begin{tabular}{|l|} 
of \\
\end{tabular} & i & 을 & 远 & $2 \%$ & $\frac{o}{z}$ & $\frac{0}{2}$ & 2 & $\frac{0}{2}$ \\
\hline F & $i_{z}^{\prime}$ & o & $\stackrel{\circ}{z}$ & $2 \frac{1}{2}$ & $\frac{o}{z}$ & $\frac{0}{z}$ & \begin{tabular}{l|}
2 \\
\end{tabular} & $\frac{0}{2}$ \\
\hline 残 & $\frac{\circ}{2}$ & $\frac{\circ}{2}$ & $\frac{o}{z}$ & 20 & $\frac{o}{2}$ & $\frac{1}{2}$ & 2 & $\frac{0}{2}$ \\
\hline 敌 & i & $\stackrel{\circ}{2}$ & 远 & ¿2: & $\frac{o}{z}$ & $\frac{0}{2}$ & 2 & $\frac{0}{2}$ \\
\hline 8 & o & $\stackrel{\circ}{z}$ & $\frac{o}{z}$ & $\begin{array}{ll}10 \\
2\end{array}$ & $\frac{o}{z}$ & $\frac{0}{z}$ & 20 & $\frac{0}{z}$ \\
\hline \begin{tabular}{|l|}
$g$ \\
\end{tabular} & $\frac{\circ}{2}$ & $\frac{\circ}{2}$ & $\frac{o}{z}$ & 20 & $\frac{o}{z}$ & $\frac{1}{2}$ & 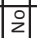 & $\frac{0}{2}$ \\
\hline F) & z & $\stackrel{\circ}{2}$ & 远 & ¿े & z & $\frac{0}{2}$ & 2 & $\frac{0}{2}$ \\
\hline 7 & o & $\stackrel{\circ}{z}$ & $\stackrel{0}{z}$ & $\frac{1}{2}=0$ & z & z & 2 & z \\
\hline g & $\frac{\circ}{2}$ & $\frac{\circ}{2}$ & $\frac{o}{z}$ & 20 & z & $\frac{1}{2}$ & \% & 잉 \\
\hline कि & 을 & z & : & ¿े & z & z & 20 & z \\
\hline \begin{tabular}{|l|} 
\\
$m$
\end{tabular} & o & zo & $\frac{0}{z}$ & $\frac{1}{2}=\frac{1}{2}$ & z & z & $\frac{0}{2}$ & z \\
\hline $\mid \begin{array}{l}\mathrm{m} \\
\end{array}$ & $\frac{o}{2}$ & $\frac{0}{2}$ & $\stackrel{\circ}{2}$ & $2 \div$ & $\frac{\circ}{2}$ & $\frac{1}{2}$ & 2 & $\frac{0}{2}$ \\
\hline \begin{tabular}{|l|} 
\\
\end{tabular} & z & z & 这 & $2 \frac{10}{2}$ & z & $\frac{o}{z}$ & 2 & $\frac{0}{z}$ \\
\hline \begin{tabular}{|l|l|} 
m \\
\end{tabular} & $z_{z}^{\prime}$ & $\frac{0}{z}$ & $\frac{0}{2}$ & $\begin{array}{lll}2 & 0 \\
2\end{array}$ & $\frac{0}{z}$ & $\frac{0}{2}$ & $2 \frac{1}{2}$ & $\frac{0}{2}$ \\
\hline 店 & $\frac{o}{2}$ & $\frac{0}{2}$ & $\stackrel{\circ}{2}$ & ¿20 & $\frac{0}{2}$ & $\frac{0}{2}$ & 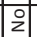 & $\frac{0}{2}$ \\
\hline m & z & z & 远 & $2 \div$ & z & $\frac{o}{z}$ & 2 & $\frac{0}{z}$ \\
\hline$\circ$ 용 & $\frac{0}{2}$ & $\frac{0}{z}$ & $\frac{0}{2}$ & $\begin{array}{ll}0 & 0 \\
2\end{array}$ & $\frac{0}{z}$ & $\frac{0}{2}$ & $\frac{0}{2}$ & $\frac{0}{2}$ \\
\hline है। & $\frac{o}{z}$ & i & $\stackrel{\circ}{2}$ & $2 \%$ & $\frac{0}{2}$ & $\frac{0}{2}$ & $\begin{array}{ll}2 \\
\end{array}$ & $\frac{0}{2}$ \\
\hline ti & z & z & $\stackrel{\circ}{z}$ & $2 \frac{1}{2}$ & z & zo & 20 & z \\
\hline 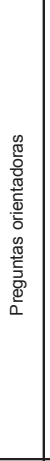 & 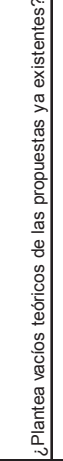 & 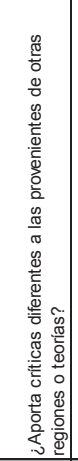 & & 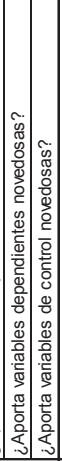 & 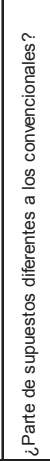 & 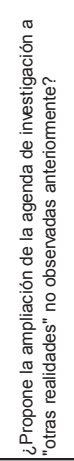 & 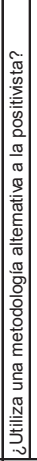 & 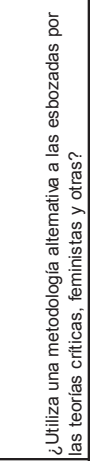 \\
\hline 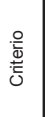 & & & & & 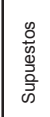 & 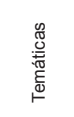 & & $\begin{array}{l}\frac{\pi}{0} \\
\frac{0}{0} \\
\frac{0}{0} \\
\frac{0}{2}\end{array}$ \\
\hline
\end{tabular}

